\title{
Guest editorial: special issue on Inductive Logic Programming
}

\author{
Stephen Muggleton · Ramon Otero $\cdot$ Simon Colton
}

Published online: 13 November 2007

Springer Science+Business Media, LLC 2007

This special issue is associated with the 16th International Conference of Inductive Logic Programming (ILP 2006), which represented a radical departure from previous years. Submissions were requested in two phases. The first phase involved submission of short papers ( 3 pages) which were then presented at the conference and included in a short papers proceedings. In the second phase, reviewers selected papers for long paper submission (15 pages maximum). These were then assessed by the same reviewers, who then decided which papers to include in the Journal Special Issue and Proceedings.

In the first phase there were a record 77 papers, compared to the usual 20 or so long papers of previous years. Each paper was reviewed by 3 reviewers. Out of these, 71 were invited to submit long papers. Out of the long paper submissions, 7 were selected for the Machine Learning Journal Special Issue and 27 were accepted for the conference proceedings. In addition, two papers were nominated by PC referees for the applications prize and two for the theory prize. The papers in this special issue represent the diversity and vitality in present ILP research.

Two of the papers are related to search in ILP. Firstly, a novel stochastic search technique is investigated in the paper, "QG/GA: A Stochastic Search for Progol" (Muggleton and Tamaddoni-Nezhad). This was a theory prize-winner, and describes a new algorithm for efficiently constructing randomly-chosen consistent hypotheses within the Progol framework. The paper presents a theoretical framework for the approach together with a time-complexity

\footnotetext{
S. Muggleton $\cdot$ S. Colton $(\bowtie)$

Computational Bioinformatics Laboratory, Department of Computing, Imperial College, 180 Queens

Gate, London SW7 2RH, UK

e-mail: sgc@doc.ic.ac.uk

S. Muggleton

e-mail:shm@doc.ic.ac.uk

R. Otero

Artificial Intelligence Laboratory, Department of Computer Science, University of Corunna, Campus de Elvina, 15071 Coruna, Spain

e-mail: otero@dc.fi.udc.es
} 
result. The approach is demonstrated on a series of benchtest problems. Next, ongoing research in the area of Phase Transition effects in ILP search is covered in "On the Connection Between the Phase Transition of the Covering Test and the Learning Success Rate in ILP" (Alphonse and Osmani). In this paper, the authors investigate the plateau phenomenon in the well-known ILP phase transition work of Giordana and Saitta (2000). It is shown that a top-down data-driven strategy can defeat any plateau when supplied with near-miss data.

Recent Probabilistic ILP research is represented in the paper "Compressing Probabilistic Prolog Programs" (De Raedt, Kersting, Kimmig and Revoredo), which describes an approach to simplifying ProbLog programs. The compression approach involves selecting a subset of clauses from a given ProbLog program which maximizes the likelihood with respect to a set of examples. Also in this general area, the paper "Generalized Ordering-search for Learning Directed Probabilistic Logical Models" (Ramon, Croonenborghs, Fierens, Blockeel and Bruynooghe) introduces an algorithm to learn both structure and conditional probability distributions of directed probabilistic models. This approach is demonstrated on various application domains.

The combination of ILP and support-vector technology is an area of increasing interest. In the second theory-prize paper "Margin-Based First-order Rule Learning" (Ruckert and Kramer) a new margin-based approach to first-order rule learning is introduced. Analysis in the paper indicates a means of optimizing the mean of the margin minus its variance which leads to an algorithm which runs in time linear in the number of examples. Experiments indicate that the approach is good at avoiding overfitting.

The paper "ALLPAD: Approximate Learning of Logic Programs with annotated Disjunctions" (Riguzzi) describes an algorithm for learning Logic Programs with Annotated Disjunctions (LPADs). The algorithm is demonstrated on a number of real and artificial problems.

Work on applying ILP to problems in Systems Biology is represented in the paper "Inductive Logic Programming for Gene Regulation Prediction" (Frohler and Kramer). The paper shows that adding functional categorization and protein-protein interactions substantially improves the performance of predicting gene regulation signals.

ILP 2006 was held in Santiago de Compostela under the auspices of the Department of Computer Science at the University of Corunna and the Grupo de Sistemas Intelixentes at the University of Santiago de Compostella. The annual meeting of ILP researchers acts as the premier forum for presenting the latest work in the field. In addition to the many technical paper presentations, the invited talks this year were given by some of the most distinguished names in Artificial Intelligence research, namely John McCarthy, Vladimir Lifschitz, Bart Selman, Ehud Shapiro and Brian Milch.

We gratefully acknowledge support of the PASCAL network of excellence, the Government of Spain, National Commissions of Science and Technology, the Government of Galicia-Spain, Secretary of Research and Design, the University of Corunna, Imperial College London, the University of Santiago de Compostela and the Spanish Association of AI.

Finally we would like to thank the many individuals involved in the preparation of the conference. These include the Conference Proceedings Organizer (Alireza TamaddoniNezhad), the local chair (David Losada), the local organizers (Jorge Gonzales and Miguel Varela) as well as Bridget Gundry who organized and distributed the conference poster. 